Revised version

\title{
Non-Linear Deformations of Liquid-Liquid Interfaces Induced by Electromagnetic Radiation Pressure
}

\author{
Aslak Hallanger*, Iver Brevik ${ }^{\dagger}$, Skjalg Haaland ${ }^{\ddagger}$ \\ Department of Energy and Process Engineering, \\ Norwegian University of Science and Technology, N-7491 Trondheim, Norway \\ Roger Sollie ${ }^{\S}$ \\ Department of Physics, \\ Norwegian University of Science and Technology, \\ N-7491 Trondheim, Norway, \\ and \\ Statoil Research Centre, \\ N-7005 Trondheim, Norway,
}

(Dated: October 25, 2018)

\footnotetext{
* Email: aslakhallanger@hotmail.com

$\dagger$ Email: iver.h.brevik@ntnu.no

‡Email: skjalg.haaland@ntnu.no

§ Email: rsol@statoil.com
} 


\begin{abstract}
The idea of working with a near-critical phase-separated liquid mixture whereby the surface tension becomes weak, has recently made the field of laser manipulation of liquid interfaces a much more convenient tool in practice. The deformation of interfaces may become as large as several tenths of micrometers, even with the use of conventional laser power. This circumstance necessitates the use of nonlinear geometrical theory for the description of surface deformations. The present paper works out such a theory, for the surface deformation under conditions of axial symmetry and stationarity. Good agreement is found with the experimental results of Casner and Delville [A. Casner and J. P. Delville, Phys. Rev. Lett. 87, 054503 (2001); Opt. Lett. 26, 1418 (2001); Phys. Rev. Lett. 90, 144503 (2003)], in the case of moderate power or a broad laser beam. In the case of large power and a narrow beam, corresponding to surface deformations of about 50 micrometers or higher, the theory is found to over-predict the deformation. Possible explanations of this discrepancy are discussed.
\end{abstract}

PACS numbers: 42.25.Gy, 42.50.Vk, 82.70.Kj 


\section{INTRODUCTION}

The formation, deformation, and breakup of fluid interfaces are ubiquitous phenomena in nature [1]. One special group of effects which implies the so-called finite time singularity 2], has as its most common example the breakup of pendant drops driven by gravity [3, 4]. If external fields such as electric or magnetic fields are present, one has in addition extra control parameters. Thus we may mention that interface instabilities driven by electric fields [5, 6] are important for many processes such as electro-spraying [7], ink-jet printing [8], or surface-relief patterning [9]. A uniform magnetic field can also be useful, for instance for the purpose of of forming elongated magnetic droplets [10]. These deformations, as well as those induced by the acoustic radiation pressure on liquid surfaces [11, 12], have been used to explore the mechanical properties of fluid interfaces in a non-contact way [13, 14].

It is noteworthy that laser-induced deformations of the interfaces of soft materials have not received the same amount of interest in the past. Most attention has been given to testparticle global effects such as optical levitation and trapping - cf., for instance, Refs. 15, 16]. The reason for this circumstance is simple: deformations of fluid interfaces by optical radiation are ordinarily weak. For instance, in the classic experiment of Ashkin and Dziedzic 17], a pulsed single transverse mode doubled Nd:YAG laser (20 pulses per second) was focused vertically from above on a water surface. The wavelength of the incident wave was $\lambda_{0}=0.53 \mu \mathrm{m}$, the peak power was $P_{\max }=3 \mathrm{~kW}$ (low enough to make nonlinear effects negligible), and the duration of each pulse was $60 \mathrm{~ns}$. The beam radius at the waist was reported to be very small, $\omega_{0}=2.1 \mu \mathrm{m}$, but has most likely have been somewhat larger, $\omega_{0}=4.5 \mu \mathrm{m}$; cf. the dicussion in Ref. [18]. The rise of the water surface was small, about $0.9 \mu \mathrm{m}$, occurring at $t \approx 450 \mathrm{~ns}$ after the onset of the pulse. The physical reason for this small surface elevation is evidently the large surface tension $\sigma=0.073 \mathrm{~N} / \mathrm{m}$ between air and water. [The theory of the Ashkin-Dziedzic experiment was worked out in Refs. 18, 19].] If we go to the more recent experiment of Sakai et al. [20], we will find that the surface displacement was even more minute. In this case the displacement was induced by a continuous wave (cw) $\mathrm{Ar}^{+}$pump laser (wavelength in vacuum $\lambda_{0}=0.514 \mu \mathrm{m}$, maximum power $P_{\max }=0.5$ $\mathrm{W})$, and was probed with a He-Ne laser. For a beam power $P=0.3 \mathrm{~W}$ and a beam waist $\omega_{0}=142 \mu \mathrm{m}$ the elevation of the surface was found to be extremely small, about $2 \mathrm{~nm}$.

Generally speaking it is of considerable interest to be able to probe the displacement of 
fluid interfaces in a way that is non-contact, i.e., which avoids a direct touch of the fluid by mechanical devices. The only acting force on the fluid surface is thus the radiation force. As discussed in Ref. [21], for instance, this kind of force can measure locally the micromechanical properties of soft biological systems because artificial membranes [22] or cells [23] can be highly deformable. Sizable effects of the radiation pressure should thus be measured, facilitating the characterization of surface elasticity properties. A major step forward was to recognize that one can reduce the surface tension considerably by working with a phase-separated liquid mixture, close to the critical point. In this way "giant" deformations can be achieved. Recent experiments of Casner and Delville have shown that the displacements can in this way reach several tenths of micrometers [21, 24, 25, 26, 27] (cf. also the recent review [28]). Also, there are seen in the experiments rather remarkable asymmetries with respect to the upward/downward direction of the laser beam [27].

The giant deformations make it necessary to use nonlinear theory for the description of the surface deflection. The purpose of the present paper is to present such a calculation, for the typical case where the elevation is axially symmetric (a condition almost always satisfied in practice), and in addition stationary. Comparison with the mentioned experiments will be made. We shall moreover assume that the laser beam is incident from below only. The mentioned up/down asymmetry will thus not be treated.

For completeness we emphasize that we are considering nonlinear theory only in a geometrical sense, in order to describe the interface bulge. The electromagnetic theory as such is kept on the conventional linear level, as we are only considering moderate laser intensities. The theory for linear deformations has been worked out before [18, 19, 21, 25]. The analytic solution for the deflection of the surface is reproduced in Eq. (47).

\section{DERIVATION OF THE GOVERNING EQUATION}

\section{A. Basic electromagnetic theory}

We begin by writing down the expression for the electromagnetic volume force density $\mathbf{f}$ in an isotropic, nonconducting and nonmagnetic medium [18, 29]:

$$
\mathbf{f}=-\frac{1}{2} E^{2} \nabla \varepsilon+\frac{1}{2} \nabla\left[E^{2} \rho\left(\frac{\partial \varepsilon}{\partial \rho}\right)_{S}\right]+\frac{\kappa-1}{c^{2}} \frac{\partial}{\partial t}(\mathbf{E} \times \mathbf{H}) .
$$


Here $\mathbf{E}$ and $\mathbf{H}$ are the electric and magnetic fields, $\rho$ is the mass density of the medium (fluid), $\varepsilon$ is the permittivity, and $\kappa=\varepsilon / \varepsilon_{0}$ is the relative permittivity where $\varepsilon_{0}$ denotes the permittivity of vacuum.

Let us comment on the various terms in Eq. (11), beginning with the last term. This term is called the Abraham term, since it follows from Abraham's electromagnetic energymomentum tensor. The term is experimentally detectable under special circumstances at low frequencies [18], but not at optical frequencies, at least not under usual stationary conditions. The Abraham term simply fluctuates out.

The middle term in Eq. (11) is the electrostriction term. When seen from within the optically denser medium (the medium with the highest $n$ ), the electrostriction force is always compressive. Whether this kind of force is detectable in a static or a stationary case, depends on whether the experiment is able to measure local pressure distributions within the compressed region or not. Moreover, in a dynamic case the velocity of sound is an important factor. If the elastic pressure in the fluid has sufficient time to build up, then the electrostriction force will not be detectable when measuring the gross behavior of a fluid such as the elevation of its surface. Such is usually the case in optics. The time required for the counterbalance to take place, is of the same order of magnitude as the time needed for sound waves to traverse the cross section of the laser beam. For a beam width around $10 \mu \mathrm{m}$, this yields a time scale for counterbalance of the order of $10 \mathrm{~ns}$. For instance, in the Ashkin-Dziedzic experiment [17] a detailed calculation verifies this time scale; cf. Fig. 9 in [18].

Another point worth mentioning in connection with the electrostriction term is that that we have written $(\partial \varepsilon / \partial \rho)_{S}$ as an adiabatic partial derivative. This seems most natural in optical problems in view of the rapid variations of the field, at least in connection with laser pulses. In many cases it is however legitimate to assume that the medium is nonpolar, so that we need not distinguish between adiabatic and isothermal derivatives. The permittivity depends on the mass density only. Then derivative can be written simply as $d \varepsilon / d \rho$, and is calculable from the Clausius-Mossotti relation. In this way we can write Eq. (11) in the following form, when omitting the last term,

$$
\mathbf{f}=-\frac{1}{2} \varepsilon_{0} E^{2} \nabla \kappa+\frac{1}{6} \varepsilon_{0} \nabla\left[E^{2}(\kappa-1)(\kappa+2)\right] .
$$

Finally, we have the first term in Eq. (11), which may be called the Abraham-Minkowski 
force, since it follows equally well from the Abraham and the Minkowski energy-momentum tensors:

$$
\mathbf{f}^{A M}=-\frac{1}{2} \varepsilon_{0} E^{2} \nabla \kappa
$$

This is the only term that we have to take into account in practice in optics, under usual circumstances. We see that this force is equal to zero in the homogeneous interior of the medium, and acts in the inhomogeneous boundary region only. By integrating the normal component of the Abraham-Minkowski force density across the boundary, we obtain the surface force density which can alternatively be evaluated as the jump of the normal component of the electromagnetic Maxwell stress tensor.

In the following we focus the attention on the force term in Eq. (3) only.

\section{B. Surface tension and radiation forces on a curved surface}

Let us assume that there is established a stationary curved surface $z=h(x, y)$ distinguishing two fluids, a lower fluid (1) and an upper fluid (2), the equilibrium position being determined by the balance of gravity, surface tension, and radiation pressure. The undisturbed position of the surface is the $x y$ plane. Because of the surface tension coefficient $\sigma$, there will be a normal stress proportional to the mean curvature of the surface:

$$
p_{2}-p_{1}=\sigma\left(\frac{1}{R_{1}}+\frac{1}{R_{2}}\right)
$$

$R_{1}$ and $R_{2}$ being the principal radii of curvature at the surface point considered. If $R_{1}$ and $R_{2}$ are positive, $p_{2}-p_{1}>0$. This means that the pressure is greater in the medium whose surface is convex. It is useful to have in mind the following general formula for the mean curvature $1 / R_{1}+1 / R_{2}$ :

$$
\frac{1}{R_{1}}+\frac{1}{R_{2}}=\frac{h_{x x}\left(1+h_{y}^{2}\right)+h_{y y}\left(1+h_{x}^{2}\right)-2 h_{x y} h_{x} h_{y}}{\left(1+h_{x}^{2}+h_{y}^{2}\right)^{3 / 2}},
$$

where $h_{x}=\partial h / \partial x$, etc. Our convention is such that the curvature is positive if the surface is concave upwards. Also, we note that the unit normal vector $\mathbf{n}$ to the surface is

$$
\mathbf{n}=\left(1+h_{x}^{2}+h_{y}^{2}\right)^{-1 / 2}\left(-h_{x},-h_{y}, 1\right)
$$

The normal points upwards, from medium 1 to medium 2. 
Assume now that there is a monochromatic electromagnetic wave with electric field vector $\mathbf{E}^{(i)}(\mathbf{r}) e^{-i \omega t}$ incident from below, in the positive $z$ direction. The direction of the incident wave vector $\mathbf{k}_{i}$ is thus given by the unit vector

$$
\hat{\mathbf{k}}_{i}=(0,0,1)
$$

in medium 1. When this wave impinges upon the surface, it becomes separated into a transmitted wave $\mathbf{E}^{(t)}$ and a reflected wave $\mathbf{E}^{(r)}$, propagating in the directions of $\hat{\mathbf{k}}_{t}$ and $\hat{\mathbf{k}}_{r}$, respectively. We assume, in conformity with usual practice, that the waves can locally be regarded as plane waves and that the surface can locally be regarded as plane. The plane of incidence is formed by the vectors $\hat{\mathbf{k}}_{i}$ and $\mathbf{n}$; we call the angle of incidence $\theta_{i}$ and the angle of transmission $\theta_{t}$. See Fig. 1. Moreover, we let $\mathbf{E}_{\|}$and $\mathbf{E}_{\perp}$ be the components of $\mathbf{E}$ parallel and perpendicular to the plane of incidence, respectively. The expressions for the energy flux transmission coefficients $T_{\|}$and $T_{\perp}$ for a plane wave incident upon a boundary surface are (cf. [29], p. 496):

$$
\begin{gathered}
T_{\|}=\frac{n_{2}}{n_{1}} \frac{\cos \theta_{t}}{\cos \theta_{i}}\left(\frac{E_{\|}^{(t)}}{E_{\|}^{(i)}}\right)^{2}=\frac{\sin 2 \theta_{i} \sin 2 \theta_{t}}{\sin ^{2}\left(\theta_{i}+\theta_{t}\right) \cos ^{2}\left(\theta_{i}-\theta_{t}\right)}, \\
T_{\perp}=\frac{n_{2}}{n_{1}} \frac{\cos \theta_{t}}{\cos \theta_{i}}\left(\frac{E_{\perp}^{(t)}}{E_{\perp}^{(i)}}\right)^{2}=\frac{\sin 2 \theta_{i} \sin 2 \theta_{t}}{\sin ^{2}\left(\theta_{i}+\theta_{t}\right)}
\end{gathered}
$$

When dealing with an unpolarized radiation field, one usually averages over the two polarizations and represents the transmission coefficient by the single entity

$$
\langle T\rangle=\frac{1}{2}\left(T_{\|}+T_{\perp}\right)
$$

Consider now the electromagnetic surface force density, which we will call $\boldsymbol{\Pi}$. As mentioned above, $\Pi$ can be found by integrating the normal component of the volume force density across the surface boundary layer. From Eq. (3) it follows that the surface force acts normal to the surface, and that it is directed towards the optically thinner medium.

We introduce the intensity $I$ of the incident beam,

$$
I=\varepsilon n_{1} c\left\langle E^{(i)^{2}}\right\rangle
$$

(in the case of azimuthal symmetry $I=I(r)$ ), and let $\alpha$ denote the angle between $\mathbf{E}^{(i)}$ and the plane of incidence,

$$
E_{\|}^{(i)}=E^{(i)} \cos \alpha, \quad E_{\perp}^{(i)}=E^{(i)} \sin \alpha
$$


Then, we can write the surface force density as

$$
\boldsymbol{\Pi}=-\frac{I}{2 c} \frac{n_{2}^{2}-n_{1}^{2}}{n_{2}} \frac{\cos \theta_{i}}{\cos \theta_{t}}\left[\left(\sin ^{2} \theta_{i}+\cos ^{2} \theta_{t}\right) T_{\|} \cos ^{2} \alpha+T_{\perp} \sin ^{2} \alpha\right] \mathbf{n} .
$$

When $\mathbf{E}^{(i)}=\mathbf{E}_{\|}^{(i)}$ or $\mathbf{E}^{(i)}=\mathbf{E}_{\perp}^{(i)}$ (i.e., $\alpha=0$ or $\pi / 2$ ) it is often convenient to express $\boldsymbol{\Pi}$ as

$$
\boldsymbol{\Pi}=\frac{n_{1} I}{c} \cos ^{2} \theta_{i}\left(1+R-\frac{\tan \theta_{i}}{\tan \theta_{t}} T\right) \mathbf{n},
$$

where $R=1-T$ is the reflection coefficient. This expression has been derived before 26, 27, 30]. It holds also in the hydrodynamic nonlinear case. In connection with the mentioned Bordeaux experiments [21, 24, 25, 26, 27] , the upper liquid was always the optically denser one. Thus $n_{2}>n_{1}$, the direction of $\boldsymbol{\Pi}$ is antiparallel to $\mathbf{n}$, and the force acts downward, normal to the surface.

The case of normal incidence yields

$$
\begin{aligned}
& T_{\|}=T_{\perp}=\frac{4 n_{1} n_{2}}{\left(n_{2}+n_{1}\right)^{2}}, \\
& \boldsymbol{\Pi}=-\frac{2 n_{1} I}{c} \frac{n_{2}-n_{1}}{n_{2}+n_{1}} \mathbf{n} .
\end{aligned}
$$

\section{Cylindrical symmetry}

We henceforth assume cylindrical symmetry, using standard cylinder coordinates $(r, \theta, z)$. There is no variation in the azimuthal direction, $\partial h / \partial \theta=0$. With the notation $h_{r}=\partial h / \partial r$ we have

$$
\cos \theta_{i}=\frac{1}{\sqrt{1+h_{r}^{2}}}, \quad \sin \theta_{i}=\frac{h_{r}}{\sqrt{1+h_{r}^{2}}} .
$$

Together with analogous expressions for $\theta_{t}$ this can be inserted into Eq. (13) to yield

$$
\Pi=-\frac{2 n_{1} I(r)}{c} \frac{1-a}{1+a} f\left(h_{r}, \alpha\right) \mathbf{n}
$$

where $a$ is the relative refractive index,

$$
a=n_{1} / n_{2}<1
$$

and $f\left(h_{r}, \alpha\right)$ is the function

$$
f\left(h_{r}, \alpha\right)=\frac{(1+a)^{2}}{\left[a+\sqrt{1+\left(1-a^{2}\right) h_{r}^{2}}\right]^{2}}
$$




$$
\times\left\{\sin ^{2} \alpha+\frac{1+\left(3-a^{2}\right) h_{r}^{2}+\left(2-a^{2}\right) h_{r}^{4}}{\left[a h_{r}^{2}+\sqrt{1+\left(1-a^{2}\right) h_{r}^{2}}\right]^{2}} \cos ^{2} \alpha\right\} .
$$

When the surface is horizontal, $h_{r}=0$, we have $f=1$, and $\Pi$ reduces to the expression (16).

A peculiar property of the expression (20) facilitating practical calculations is that it is quite insensitive with respect to variations in the polarization angle $\alpha$, especially in the case when $a$ is close to unity, which is in practice most important. Thus if we draw curves for $\Pi\left(\theta_{i}\right)$ versus $\theta_{i}$ for various input values of $\alpha$ in the whole region $0<\alpha<90^{\circ}$ (not shown here), we will find that the curves lie close to each other. For practical calculations involving unpolarized light it is thus legitimate to replace $f\left(h_{r}, \alpha\right)$ by its average with respect to $\alpha$. As $\left\langle\sin ^{2} \alpha\right\rangle=\left\langle\cos ^{2} \alpha\right\rangle=1 / 2$, we can then write the surface force density as

$$
\boldsymbol{\Pi}=-\frac{2 n_{1} I(r)}{c} \frac{1-a}{1+a} f\left(h_{r}\right) \mathbf{n},
$$

where $f\left(h_{r}\right)$ is equal to $f\left(\alpha, h_{r}\right)$ averaged over $\alpha$,

$$
f\left(h_{r}\right)=(1+a)^{2} \frac{1+\left(2-a^{2}\right) h_{r}^{2}+a h_{r}^{2} \sqrt{1+\left(1-a^{2}\right) h_{r}^{2}}+h_{r}^{4}}{\left[a+\sqrt{1+\left(1-a^{2}\right) h_{r}^{2}}\right]^{2}\left[a h_{r}^{2}+\sqrt{1+\left(1-a^{2}\right) h_{r}^{2}}\right]^{2}} .
$$

This expression is valid also in the case of hydrodynamic nonlinearity. Note again that $\Pi$ is the normally-directed force per unit area of the oblique liquid surface.

Finally, let us consider the force balance for the liquid column, assuming stationary conditions. When $n_{2}>n_{1}$ the surface tension force which acts upward, has to balance the combined effect of gravity and electromagnetic surface force, which both act downward. When the surface is given as $h=h(r, \theta)$, the mean curvature can be written as

$$
\frac{1}{R_{1}}+\frac{1}{R_{2}}=\frac{1}{r} \frac{\partial}{\partial r} \frac{r h_{r}}{\sqrt{1+h_{r}^{2}+\left(h_{\theta} / r\right)^{2}}}+\frac{1}{r^{2}} \frac{\partial}{\partial \theta} \frac{h_{\theta}}{\sqrt{1+h_{r}^{2}+\left(h_{\theta} / r\right)^{2}}},
$$

with sign conventions the same as in Eq. (15). Thus for azimuthal symmetry,

$$
\frac{1}{R_{1}}+\frac{1}{R_{2}}=\frac{1}{r} \frac{d}{d r} \frac{r h_{r}}{\sqrt{1+h_{r}^{2}}}
$$

and the force balance becomes [26, 31]

$$
\left(\rho_{1}-\rho_{2}\right) g h(r)-\frac{\sigma}{r} \frac{d}{d r}\left[\frac{r h_{r}}{\sqrt{1+h_{r}^{2}}}\right]=\Pi(r) .
$$


This equation follows from considering the equilibrium of a liquid column having unit base area. Here $\Pi(r)$ is the pressure scalar, i.e., $\Pi(r)=\Pi(r) \mathbf{n}$. Thus $\Pi(r)<0$.

What expression to insert for $\Pi(r)$ in Eq. (25), depends on the physical circumstances. Thus in the case of an unpolarized laser beam, we may use either the expression (14) with $R=\langle R\rangle, T=\langle T\rangle$, or alternatively use the expression (21). We will follow the latter option here. As noted, there is no restriction imposed on the magnitude of the slope of the surface.

\section{SOLUTION OF THE NONLINEAR EQUATION}

It is advantageous to introduce nondimensional variables. Let us first define the capillary length $l_{C}$ and the Bond number $B$,

$$
l_{C}=\sqrt{\frac{\sigma}{\left(\rho_{1}-\rho_{2}\right) g}}, \quad B=\left(\frac{\omega_{0}}{l_{C}}\right)^{2},
$$

$\omega_{0}$ being the radius of the beam waist. The Bond number describes the strength of buoyancy relative to the Laplace force. If $B \ll 1$, gravity is much weaker than the Laplace force. (The Bordeaux experiments covered the region $10^{-3}<B<4$.) We then define the nondimensional radius $R$ and the nondimensional height $H(R)$ as

$$
R=\frac{r}{\omega_{0}}, \quad H(R)=\frac{h(r)}{l_{C}} .
$$

The fact that in practice $a=n_{1} / n_{2}$ is very close to one, makes it at first sight possible to simplify the right hand side of the governing equation (25). Namely, from Eq. (22) one would expect that $f\left(h_{r}\right) \rightarrow 1$. However, the situation is more delicate due to nonlinearity: if we keep $f\left(h_{r}\right)$ in the formalism and calculate the elevation $h(r)$, we will find that $f$ gets a pronounced dip within the region where the beam is strong. Typically, if we draw a curve for $f=f(R)$ versus the nondimensional radius $R$, we will see that $f$ drops from 1 to about 0.3 when $R$ lies about 0.5 . Mathematically, this is because the high steepness of the surface makes $h_{r}$ (or $H_{R}$ ) large enough to influence $f$ significantly in a narrow region even when $a$ is close to unity. Assuming a Gaussian incident beam,

$$
I(r)=\frac{2 P}{\pi \omega_{0}^{2}} e^{-2 r^{2} / \omega_{0}^{2}}
$$

with $P$ the beam power, we may write the governing equation (25) as a nonlinear differential equation for $H$ :

$$
B H-\frac{H_{R R}+\frac{1}{R} H_{R}+\frac{1}{B R} H_{R}^{3}}{\left(1+\frac{1}{B} H_{R}^{2}\right)^{3 / 2}}=-F e^{-2 R^{2}} f\left(H_{R}\right) .
$$


Here $F$ is a positive constant at fixed temperature,

$$
F=\frac{2\left(n_{2}-n_{1}\right) P}{\pi c g\left(\rho_{1}-\rho_{2}\right) l_{C}^{3}},
$$

which can for practical purposes be written as, since $(\partial n / \partial \rho)_{T}=-1.22 \times 10^{-4} \mathrm{~m}^{3} / \mathrm{kg}$,

$$
F=\frac{2}{\pi c g}\left(-\frac{\partial n}{\partial \rho}\right)_{T} \frac{P}{l_{C}^{3}}=26400 \frac{P}{l_{C}^{3}} .
$$

In the last equation, the dimension of $l_{C}$ is $\mu \mathrm{m}$.

The two quantities $l_{C}$ and $a$ will vary with the temperature $T$ in accordance with the theory of critical phenomena. Thus for the density contrast $\Delta \rho=\rho_{1}-\rho_{2}$ we have

$$
\Delta \rho=(\Delta \rho)_{0}\left(\frac{T-T_{C}}{T_{C}}\right)^{\beta},
$$

where $\beta=0.325,(\Delta \rho)_{0}=285 \mathrm{~kg} / \mathrm{m}^{3}, T_{C}=308.15 \mathrm{~K}$ being the critical temperature above which the mixture separates into two different phases. Similarly

$$
\sigma=\sigma_{0}\left(\frac{T-T_{C}}{T_{C}}\right)^{2 \nu}
$$

with $\nu=0.63, \sigma_{0}=1.04 \times 10^{-4} \mathrm{~N} / \mathrm{m}$. More details can be found in Refs. [21, 25]. We give here the practically useful formulas for $a$ and $l_{C}$ :

$$
\begin{aligned}
& a=1-0.0238\left(\frac{T-T_{C}}{T_{C}}\right)^{0.325}, \\
& l_{C}=193\left(\frac{T-T_{C}}{T_{C}}\right)^{0.468}(\mu \mathrm{m}) .
\end{aligned}
$$

These two quantities are the only parameters that vary with temperature. There are thus three parameters in all in the problem, namely $T$, the beam power $P$, and the beam waist $\omega_{0}$. Nondimensionally, the last two parameters correspond to $F$ and $B$, Eqs. (31) and (26).

\section{A. Numerical solution}

It is convenient to let $H$ be positive downwards, so that in the formalism below we will replace $H$ with $-H$.

We start from the nondimensional governing equation in the form

$$
\frac{1}{R} \frac{d}{d R}\left(\frac{R H_{R}}{\sqrt{1+H_{R}^{2} / B}}\right)-B H=-F e^{-2 R^{2}} f\left(H_{R}\right),
$$


with boundary conditions

$$
H_{R}(0)=0, \quad H(\infty)=0 .
$$

We use a two-point method to solve the nonlinear differential equation iteratively. Define

$$
K=\sqrt{1+H_{R}^{2} / B}, \quad S=F e^{-2 R^{2}} f\left(H_{R}\right)
$$

and let $G=H_{R} / K$. We obtain the following first order system:

$$
\begin{gathered}
\frac{d H}{d R}=K G, \\
\frac{d G}{d R}+\frac{G}{R}-B H=-S,
\end{gathered}
$$

with boundary conditions

$$
G(0)=0, \quad H(\infty)=0
$$

We linearize the equations by means of lagging, i.e., we use values for $H_{R}$ from the last iteration in the nonlinear functions $K$ and $S$. Using a nonuniform grid with $n$ grid points, we integrate the equations between two grid points $j$ and $j+1$, letting $m$ be the midpoint and $\Delta R_{j}$ the distance between the points. We obtain

$$
\begin{gathered}
H_{j+1}-H_{j}=K_{m} \frac{\Delta R_{j}}{2}\left(G_{j}+G_{j+1}\right), \\
G_{j+1}-G_{j}+\frac{1}{R_{m}} \frac{\Delta R_{j}}{2}\left(G_{j}+G_{j+1}\right)-B \frac{\Delta R_{j}}{2}\left(H_{j}+H_{j+1}\right)=-\Delta R_{j} S_{m} .
\end{gathered}
$$

Here $H_{R}$ in $K_{m}$ and $S_{m}$ are evaluated as

$$
H_{R}=\frac{\bar{H}_{j+1}-\bar{H}_{j}}{\Delta R_{j}}
$$

where the $\bar{H}$ 's are values from the previous iteration. With $n$ grid points there are $n-1$ intervals and $n-1$ sets of equations. This confirms with the fact that there are $2 n$ quantities $H$ and $G$; since there are two boundary conditions there remain $2 n-2$ equations in all.

To start the iterations we give initial values for $H=$ const. $e^{-R^{2}}$. To deal with the boundary condition at infinity, we need in practice to replace "infinity" with a finite upper limit $R=R_{e}$. The solution falls off quite slowly with $R$, so to use the naive condition $H\left(R_{e}\right)=0$ would require $R_{e}$ to be very large. To avoid calculating the long tail of the 
solution, we can find a better boundary condition by using the lowest order term in an asymptotic expansion for $H$. When $R$ is large, $S \sim e^{-2 R^{2}}$, and $H_{R}^{2}$ is very small so that $S \approx 0, \sqrt{1+H_{R}^{2} / B} \approx 1$. Equation (40) becomes

$$
\frac{1}{R} \frac{d}{d R}\left(R H_{R}\right)-B H=0 .
$$

To lowest order this equation has the asymptotic solution $H \sim R^{-1 / 2} e^{-\sqrt{B} R}$, which in turn implies that

$$
G=H_{R}=-\left(\frac{1}{2 R}+\sqrt{B}\right) H .
$$

We take this condition to replace the condition $H=0$ at $R=R_{e}$.

We solved the discretized equations using a Block-Bidiagonal-Matrix-Algorithm, developed by one of the authors (S.H.). Our programming language was MATLAB.

\section{B. Results}

First, the following question naturally arises: at which powers $P$ will the nonlinear correction begin to be important? And what magnitudes of the centerline deformation $(r=0)$ does this correspond to? To get insight into this issue we constructed a number of figures (not shown here) for the surface height $h(r)$ versus $r$, for various temperature differences $T-T_{C}$ and beam radii $\omega_{0}$, and for various values of $P$. For each parameter set we made the calculation in two ways, viz. when taking the nonlinear correction into account, and when omitting it. Of course, there is a transitional region and no sharp limit distinguishing the linear and nonlinear regions. Our conclusions, based upon visual inspection of the curves, were that under normal conditions the linear region can be taken to prevail until $P \approx 200$ $\mathrm{mW}$. When $P>300 \mathrm{~mW}$, nonlinear effects turn up. Generally, the nonlinear deformations are higher than the linear ones. (To give an example: at $P=220 \mathrm{~mW}$ the nonlinear centerline deformation was found to be only $2 \%$ higher than the linear deformation, whereas at $P=330 \mathrm{~mW}$ it was $5 \%$ higher .)

From Refs. [21, 25] we recall that in the linear regime we have the following simple formula for the centerline height $h^{\operatorname{lin}}(r)$ (here in physical variables):

$$
h^{l i n}(r)=\frac{P}{2 \pi c g}\left(\frac{\partial n}{\partial \rho}\right)_{T} \int_{0}^{\infty} \frac{k d k}{1+k^{2} l_{C}^{2}} e^{-k^{2} \omega_{0}^{2} / 8} J_{0}(k r) .
$$

In the following we show three figures, each of them corresponding to given values of $T-T_{C}$ and $\omega_{0}$. Each figure is based upon a full nonlinear calculation. First, Fig. 2 shows how 
$h(r)$ varies with $r$ when $T-T_{C}=2.5 \mathrm{~K}$ and $\omega_{0}=4.8 \mu \mathrm{m}$. According to Eqs. (35), (26) this corresponds to $l_{C}=20.3 \mu \mathrm{m}, B=0.0560$. The powers are $P=\{300,600,1200\} \mathrm{mW}$. As mentioned above, we had to replace "infinity" with a finite outer limit $R_{e}$. Numerical trials observing the sensitivity of calculated centerline deformations showed that the choice $R_{e}=9$ was large enough. (For instance, an increase of $R_{e}$ from 9 to 10 would lead only to minute differences, the first three digits in the centerline deformation being the same.) Because of the cylindrical symmetry, only one half of the displacements $(r>0$ in the figure) need to be shown. It is seen that both powers $600 \mathrm{~mW}$ and $1200 \mathrm{~mW}$ lead to deformations much greater than $20 \mu \mathrm{m}$, and are clearly in the nonlinear region. The theoretical deflections for the three given values of the power are read off from the figure to be $\{12,56,112\} \mu \mathrm{m}$, respectively.

Our choice of input parameters makes the figure directly comparable to Fig. 6.1 in Casner's thesis 24]. The experimental centerline displacements estimated from the photos are about $10 \mu \mathrm{m}$ for $P=300 \mathrm{~mW}, 45 \mu \mathrm{m}$ for $P=600 \mathrm{~mW}$, and $75 \mu \mathrm{m}$ for $P=1200 \mathrm{~mW}$. The theoretical predictions are thus in this case larger than the observed ones, especially for the highest value of $P$. Moreover, one difference which is most noticeable is the absence of the observed "shoulder" in the theoretical solution in the case of large $P$. The shoulder occurs experimentally when the laser illumination is from below. There is at present no theory capable of describing this phenomenon. Mathematically, the establishment of the shoulder seems to be related to an instability; the real deflection jumps from one class of solutions of the nonlinear differential equation to another class. Video records actually show "jumps" in the surface when it gets formed, thus supporting our conjecture about an instability phenomenon. As for the observed width of the surface displacement, there is good agreement with the theoretical prediction.

Fig. 3 shows analogous results for the case $T-T_{C}=2.5 \mathrm{~K}, \omega_{0}=8.9 \mu \mathrm{m}$, thus a considerably broader beam. Here $l_{C}=20.3 \mu \mathrm{m}, B=0.193$. The powers are $P=\{360,600,890\}$ $\mathrm{mW}$. In this case, the value $R_{e}=7$ was found to be sufficient, for the same reasons as above. Our results can be compared with Fig. 6.3 in Casner's thesis [24]. The theoretical centerline displacements for the three mentioned cases of $P$ are $\{10,19,47\} \mu \mathrm{m}$, which all agree well with the observed values. Also in this case there occurs a shoulder experimentally, but it is not so pronounced as in the previous case.

Finally, in Fig. 4 we show the case $T-T_{C}=3 \mathrm{~K}, \omega_{0}=5.3 \mu \mathrm{m}$, corresponding to $l_{C}=22.1$ 
$\mu \mathrm{m}, B=0.0576$, for powers $P=\{300,590,830\} \mathrm{mW}$. Again, the outer nondimensional radius $R_{e}=9$ was found to be appropriate. The theoretical centerline deflections are now seen from the figure to be $\{10,39,65\} \mu \mathrm{m}$, respectively, for the given values of $P$. We may compare this with the photos shown in Fig. 2 in Ref. 27] or Fig. VI.5 in [28]: the corresponding experimental dispacements are about $\{10,40,55\} \mu \mathrm{m}$. Also this time we see that the agreement between theory and experiment is good for low powers, but that the theory over-predicts the displacement when the power gets large. The last-mentioned effect is generally most pronounced when the radius of the laser beam is small.

\section{SUMMARY, AND FINAL REMARKS}

The "giant" deformations of fluid interfaces recently obtained in the experiments of Casner and Delville [21, 24, 25, 26, 27, 28] with the use of moderate laser beam powers $P(P$ typically lying between 500 and $1000 \mathrm{~mW}$ ) have accentuated the need of taking into account nonlinear geometrical effects in the theoretical description of the interface deformation. As a rule of thumb, inferred from a visual inspection of the figures, nonlinear effects are expected to be appreciable when the deformations become larger than about $15 \mu \mathrm{m}$. When the radius $\omega_{0}$ of the laser beam is small, typically $\omega \sim 5 \mu \mathrm{m}$, a power $P \sim 1$ watt may easily lead to deflections around $100 \mu \mathrm{m}$. The physical reason for the occurrence of giant deformations is the lowering of surface tension caused by working with a phase-separated liquid mixture close to the critical point.

The nonlinear governing equation in nondimensional form can be taken as in Eq. (29) or, what was found more convenient, as in Eq. (36) where $G=H_{R} / K$ is considered as the dependent variable. We solved the set of equations (39)-(41) numerically. As a consistency

check, we made also an analogous calculation starting from Eq. (29), and got the same results. Figures 2-4 show some examples of our calculations; these are all directly comparable with the Casner-Delville experiment.

Some general conclusions that can be made from our calculations, are the following:

1. For given values of $T-T_{C}$ and $\omega_{0}$, a larger $P$ causes a larger deformation.

2. For a given $T-T_{C}$, a smaller $\omega_{0}$ causes a larger and narrower deformation.

3. For a given $\omega_{0}$, a smaller $T-T_{C}$ causes a larger and narrower deformation.

4. Very large beam waists $\left(\omega_{0} \sim 20-30 \mu \mathrm{m}\right)$ are not able to cause a nonlinear deformation, 
not even for the largest $P$ and smallest $T-T_{C}$ investigated in the Casner-Delville experiment.

5. For small $T-T_{C}$ and small $\omega_{0}$ (for instance $T-T_{C}=2.5 \mathrm{~K}$ and $\omega_{0}=4.5 \mu \mathrm{m}$ ), a power of $300 \mathrm{~mW}$ is not enough to cause a nonlinear deformation. However, a further decrease in temperature, such as to the value $T-T_{C}=1.5 \mathrm{~K}$, will take also the $300 \mathrm{~mW}$-induced deformation into the nonlinear regime.

All the items listed above are expected on physical grounds. A large incident power concentrated on a narrow cross section means a large electromagnetic field intensity and thus a large surface force. The enhanced deformation for small $T-T_{C}$ is due to the fact that the restoring buoyancy force $(\sim \Delta \rho)$ and Laplace force $(\sim \sigma)$ vanish at $T=T_{C}$; cf. Eqs. (32) and (33).

Concretely, when comparing our results with the Casner-Delville observations, we find that for broad beams the agreement between theory and experiment is quite good; cf. our discussion of Fig. 3 above. There is however a considerable theoretical over-prediction of the deflection in the case of narrow beams and high powers. Most strikingly, this is shown in the case $\omega_{0}=4.8 \mu \mathrm{m}, P=1200 \mathrm{~mW}$, as discussed in connection with Fig. 2. The physical reason for this discrepancy is not known. It may be related to the production of heat in the liquid in the presence of the strong field, or to the loss of radiation energy because of scattering from the non-avoidable corrugations on the liquid interface. Perhaps the most intriguing possibility is that the discrepancy is related to the reflection of radiation energy from the interface "shoulder", which is seen to be produced in strong fields when the illumination is from below. This effect is most likely related to an instability; the system decides to switch from one class of solutions of the nonlinear governing equation to another class. To our knowledge, no explanation exists of this effect.

[1] M. Moseler and U. Landman, Science 289, 1165 (2000).

[2] J. Eggers, Rev. Mod. Phys. 69, 865 (1997).

[3] I. Cohen, M. P. Brenner, J. Eggers, and S. R. Nagel, Phys. Rev. Lett. 83, 1147 (1999).

[4] A. U. Chen, P. K. Notz, and O. A. Basaran, Phys. Rev. Lett. 88, 174501 (2002).

[5] G. I. Taylor, Proc. R. Soc. Lond. A 313, 453 (1969).

[6] L. Oddershede and S. Nagel, Phys. Rev. Lett. 85, 1234 (2000). 
[7] A. M. Ganán-Calvo, J. Dávila, and A. Barrero, J. Aerosol Sci. 28, 249 (1997).

[8] R. Badie and D. Frits de Lange, Proc. R. Soc. Lond. A 453, 2573 (1997).

[9] E. Schäffer, T. Thurn-Albrecht, T. P. Russell, and U. Steiner, Nature 403, 874 (2000).

[10] J. C. Bacri and D. Salin, J. Magn. Magn. Mater. 39, 48 (1983).

[11] G. Hertz and H. Mende, Z. Physik 114, 354 (1939).

[12] S. A. Elrod, B. Hadimioglu, B. T. Khuri-Yakub, E. G. Rawson, E. Richley, C. F. Quate, N. N. Mansour, and T. S. Lundgren, J. Appl. Phys. 65, 3441 (1989).

[13] C. Cinbis, N. N. Mansour, and B. T. Khuri-Yakub, J. Acoustic Soc. Am. 94, 2365 (1993).

[14] C. Flament, S. Lacis, J. C. Bacri, A. Cebers, S. Neveu, and R. Perzynski, Phys. Rev. E 53, 4801 (1996).

[15] A. Ashkin, Proc. Natl. Acad. Sci. USA 94, 4853 (1997).

[16] R. Gussgard, T. Lindmo, and I. Brevik, J. Opt. Soc. Am. B 9, 1922 (1992).

[17] A. Ashkin and J. M. Dziedzic, Phys. Rev. Lett. 30, 139 (1973).

[18] I. Brevik, Phys. Reports 52, 133 (1979).

[19] H. M. Lai and K. Young, Phys. Rev. A 14, 2329 (1976).

[20] K. Sakai, D. Mizuno, and K. Takagi, Phys. Rev. E 63, 046302 (2001).

[21] A. Casner and J. P. Delville, Phys. Rev. Lett. 87, 054503 (2001).

[22] R. Bar-Ziv, E. Moses, and P. Nelson, Biophys. J. 75, 294 (1998).

[23] J. Guck, R. Ananthakrishnan, T. J. Moon, C. C. Cunningham, and J. Käs, Phys. Rev. Lett. 84, 5451 (2000).

[24] A. Casner, PhD thesis, Université Bordeaux 1, Bordeaux, France, 2001. Available at the URL address http: //tel.ccsd.cnrs.fr/documents/archives0/00/00/16/37/index.html.

[25] A. Casner and J. P. Delville, Opt. Lett. 26, 1418 (2001).

[26] A. Casner and J. P. Delville, Phys. Rev. Lett. 90, 144503 (2003).

[27] A. Casner, J. P. Delville, and I. Brevik, J. Opt. Soc. Am. B 20, 2355 (2003).

[28] J. P. Delville, A. Casner, R. Wunenburger, and I. Brevik, arXiv:physics/0407008.

[29] J. A. Stratton, Electromagnetic Theory (McGraw-Hill, New York, 1941).

[30] G. N. Borzdov, J. Math. Phys. 34, 3162 (1993).

[31] S. Mitani and K. Sakai, Phys. Rev. E 66, 031604 (2002). 


\section{Figures}

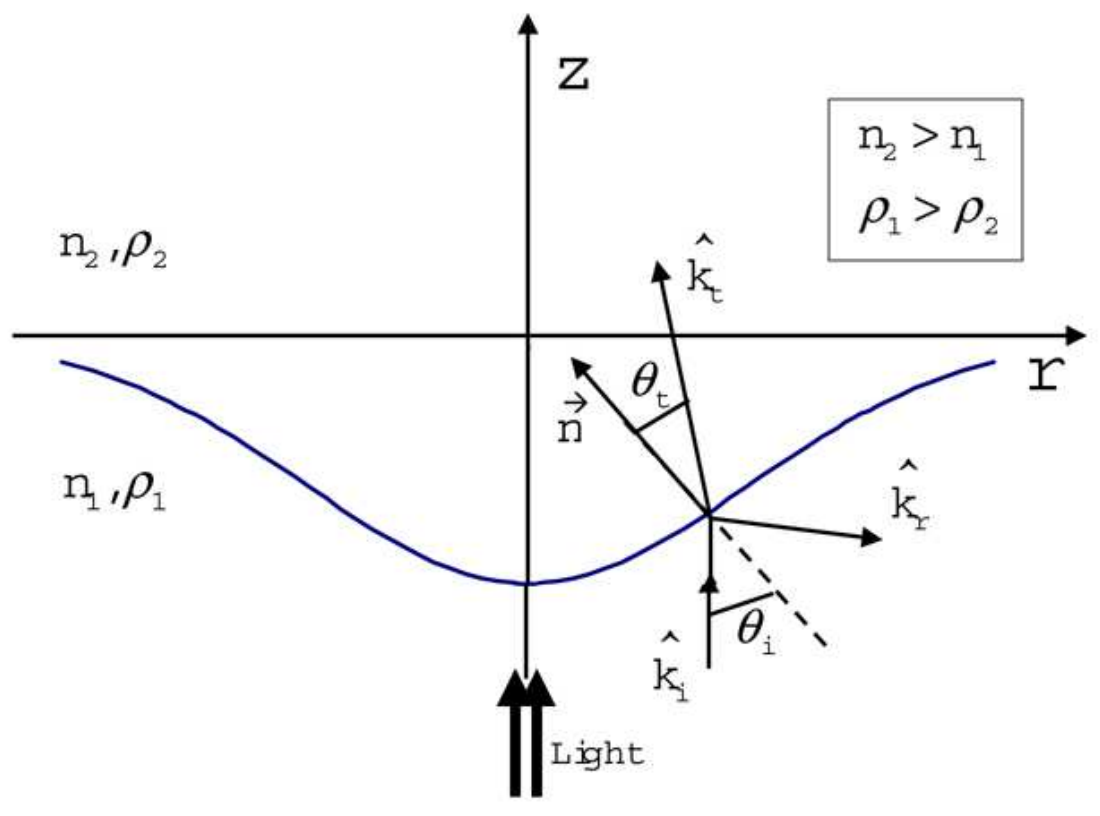

FIG. 1: Definition sketch of the displaced surface. The laser illumination is from below.

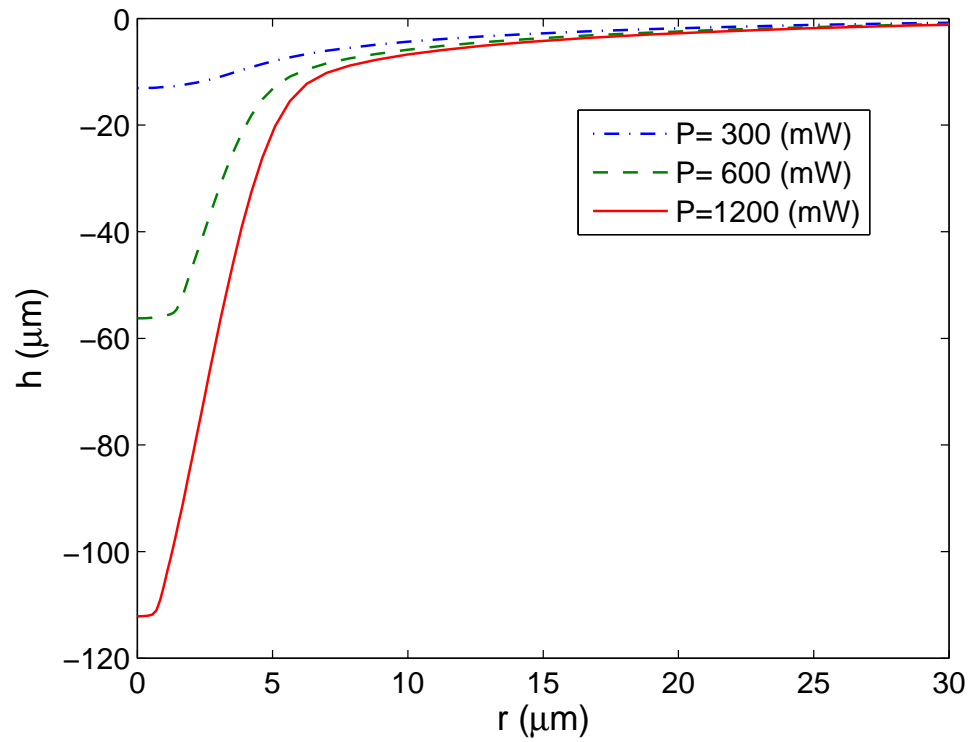

FIG. 2: Theoretical height $h(r)$ of displaced surface versus radius $r$ when $T-T_{C}=2.5 \mathrm{~K}, \omega_{0}=4.8$ $\mu \mathrm{m}$, for three different laser powers $P$. The undisturbed surface is at $h(r)=0$. 


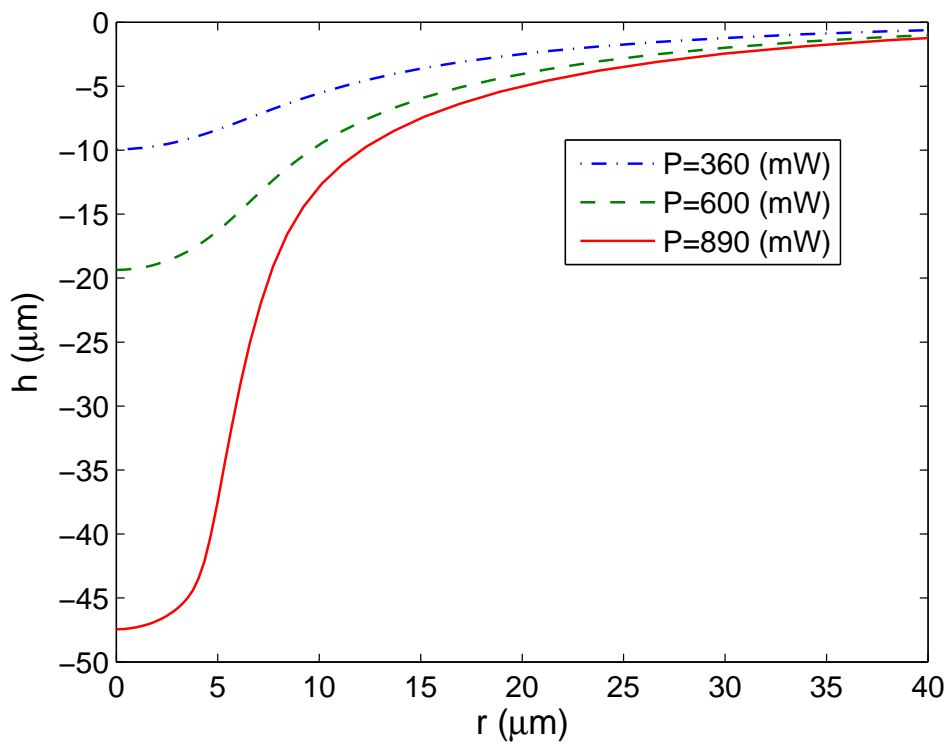

FIG. 3: Same as Fig. 2, but with $T-T_{C}=2.5 \mathrm{~K}, \omega_{0}=8.9 \mu \mathrm{m}$.

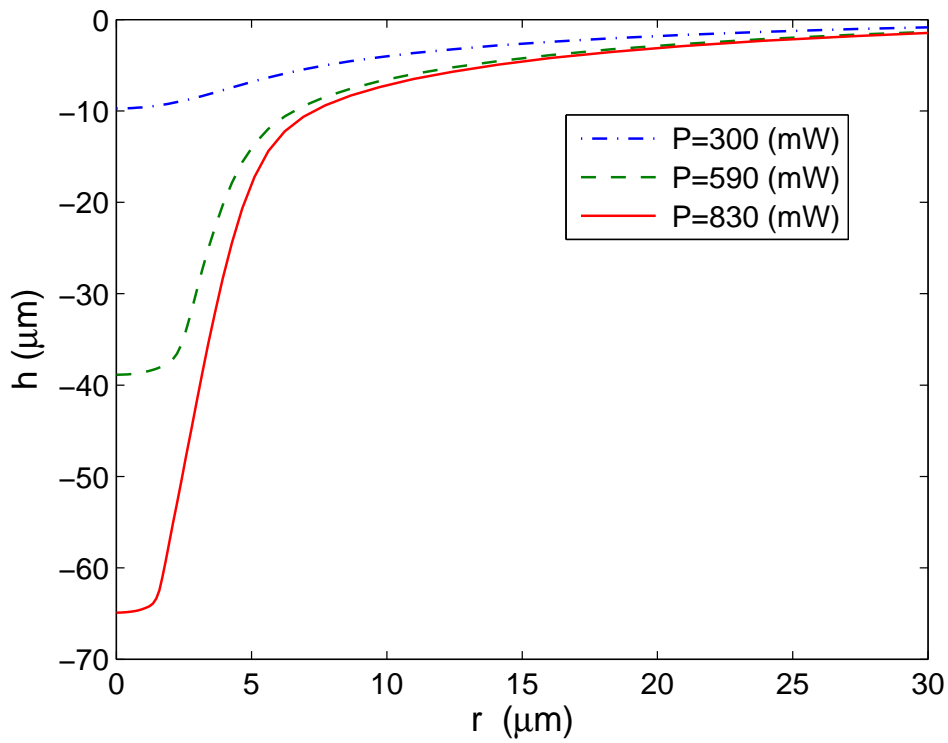

FIG. 4: Same as Fig. 2, but with $T-T_{C}=3 \mathrm{~K}, \omega_{0}=5.3 \mu \mathrm{m}$. 